\title{
HARNACK INEQUALITY FOR NON-SELF-ADJOINT EVOLUTION EQUATIONS
}

\author{
SHING-TUng YAU
}

Many years ago, S. Y. Cheng, P. Li and myself [1] gave an estimate of the heat kernel for the Laplacian. This was later improved by P. Li and myself in [2]. The key point of this later paper was a parabolic Harnack inequality that generalized an elliptic Harnack inequality that I developed more than twenty years ago.

Since many evolution equations in applied mathematics are not selfadjoint and have a convection term, I propose here to generalize the parabolic Harnack inequality that Li and I developed to cover the non-selfadjoint case. The Harnack inequality is considerably stronger than the estimates of the heat kernel. I hope that it will be useful for questions in control theory and fluid dynamics. The applications will be studied on a later occasion.

\section{Harnack inequality for non-self-adjoint equation}

Let $u$ be a positive solution of the equation

$$
\frac{\partial u}{\partial t}=\Delta u+\sum f_{i} u_{i}+V u
$$

where $f_{i}$ and $V$ may depend on $t$.

Let $\varphi=-\log u$. Then

$$
\varphi_{t}=\Delta \varphi-|\nabla \varphi|^{2}+\sum f_{i} \varphi_{i}+V
$$

Let

$$
\psi=\varphi_{t}+|\nabla \varphi|^{2}-\sum f_{i} \varphi_{i}-V-\alpha(t) \sqrt{|\nabla \varphi|^{2}+\beta(t)}-c
$$

Received June 16, 1995.

Research supported by NSF Grant DMS-92-06938. 
where $\alpha, \beta, c$ are to be chosen later.

Then

$$
\begin{aligned}
\psi_{t}= & \varphi_{t t}+2 \sum \varphi_{j} \varphi_{j t}-\sum\left(f_{i}\right)_{t} \varphi_{i}-\sum f_{i} \varphi_{t i}-V_{t} \\
& -\alpha^{\prime} \sqrt{|\nabla \varphi|^{2}+\beta}-\frac{\alpha \sum \varphi_{i} \varphi_{t i}}{\sqrt{|\nabla \varphi|^{2}+\beta}}-\frac{\alpha \beta^{\prime}}{\sqrt{|\nabla \varphi|^{2}+\beta}}-c_{t} \\
\Delta \psi= & (\Delta \varphi)_{t}+2 \sum \varphi_{i j}^{2}+2 \sum \varphi_{j}(\Delta \varphi)_{j} \\
& +2 \sum R_{i j} \varphi_{i} \varphi_{j}-\sum R_{i j} f_{i} \varphi_{j} \\
& -2 \sum f_{i, j} \varphi_{i j}-\sum f_{i}(\Delta \varphi)_{i}-\sum\left(\Delta f_{i}\right)_{i} \\
& -\Delta V-\frac{\alpha \sum \varphi_{i j}^{2}}{\sqrt{|\nabla \varphi|^{2}+\beta}}+\frac{\alpha \sum_{j}\left(\sum_{i} \varphi_{i} \varphi_{j i}\right)^{2}}{\left(|\nabla \varphi|^{2}+\beta\right)^{3 / 2}} \\
& -\frac{\alpha \sum \varphi_{i}(\Delta \varphi)_{i}}{\sqrt{|\nabla \varphi|^{2}+\beta}}-\Delta c
\end{aligned}
$$

where $R_{i j}$ is the Ricci curvature of the manifold (which is zero if defined on Euclidean space).

Hence

$$
\begin{aligned}
\psi_{t}-\Delta \psi= & -\left(|\nabla \varphi|^{2}-\sum f_{i} \varphi_{i}-V\right)_{t} \\
& -2 \sum \varphi_{i j}^{2}-2 \sum \varphi_{j}\left[|\nabla \varphi|^{2}-\sum f_{i} \varphi_{i}-V\right]_{j} \\
& +2 \sum f_{i, j} \varphi_{i j}+\sum_{i} f_{i}\left[|\nabla \varphi|^{2}-\sum f_{j} \varphi_{j}-V\right]_{i} \\
& +\sum_{i}\left[-\left(f_{i}\right)_{t}+\Delta f_{i}\right] \varphi_{i}-2 \sum R_{i j} \varphi_{i} \varphi_{j} \\
& +\sum_{i j} f_{i} \varphi_{j}+\Delta V-V_{t} \\
& +\frac{\alpha \sum_{i} \varphi_{i}\left[|\nabla \varphi|^{2}-\sum f_{j} \varphi_{j}-V\right]_{i}}{\sqrt{|\nabla \varphi|^{2}+\beta}} \\
& +\frac{\alpha \sum \varphi_{i j}^{2}}{\sqrt{|\nabla \varphi|^{2}+\beta}}-\frac{\alpha \sum \sum_{i}\left(\sum_{j} \varphi_{j i}\right)^{2}}{\left(|\nabla \varphi|^{2}+\beta\right)^{3 / 2}} \\
& -\alpha^{\prime} \sqrt{|\nabla \varphi|^{2}+\beta}-\frac{\alpha \beta^{\prime}}{2 \sqrt{|\nabla \varphi|^{2}+\beta}}-c_{t}+\Delta c
\end{aligned}
$$




$$
\begin{aligned}
= & -2 \sum \varphi_{i} \psi_{i}-2 \alpha \sum \varphi_{i}\left(\sqrt{|\nabla \varphi|^{2}+\beta}\right)_{i}-2 \sum \varphi_{i} c_{i} \\
& +\sum f_{i} \psi_{i}+\alpha \sum f_{i}\left(\sqrt{|\nabla \varphi|^{2}+\beta}\right)_{i}+\sum f_{i} c_{i} \\
& -2 \sum \varphi_{i j}^{2}+2 \sum f_{i, j} \varphi_{i j}+\sum \Delta f_{i} \varphi_{i} \\
& +\Delta V+\alpha \frac{\sum_{i} \varphi_{i}\left[|\nabla \varphi|^{2}-\sum f_{j} \varphi_{j}-V\right]_{i}}{\sqrt{|\nabla \varphi|^{2}+\beta}} \\
& -2 \sum R_{i j} \varphi_{i} \varphi_{j}+\sum R_{i j} f_{i} \varphi_{j}+\frac{\alpha \sum \varphi_{i j}^{2}}{\sqrt{|\nabla \varphi|^{2}+\beta}} \\
- & \frac{\alpha \sum\left(\sum_{j} \varphi_{j} \varphi_{j i}\right)^{2}}{\left(|\nabla \varphi|^{2}+\beta\right)^{3 / 2}}-\alpha^{\prime} \sqrt{|\nabla \varphi|^{2}+\beta}-\frac{\alpha \beta^{\prime}}{2 \sqrt{|\nabla \varphi|^{2}+\beta}}-c_{t}+\Delta c \\
= & -2 \sum \varphi_{i} \psi_{i}+\sum f_{i} \psi_{i}-2 \sum \varphi_{i j}^{2}+2 \sum f_{i, j} \varphi_{i j}+\sum\left(\Delta f_{i}\right) \varphi_{i}+\Delta V \\
& -\frac{\left(\alpha \sum f_{i, j} \varphi_{i} \varphi_{j}+\alpha \sum \varphi_{i} V_{i}\right)}{\sqrt{|\nabla \varphi|^{2}+\beta}-2 \sum R_{i j} \varphi_{i} \varphi_{j}} \\
& +\sum R_{i j} f_{i} \varphi_{j}+\frac{\alpha \sum \varphi_{i j}^{2}}{\sqrt{|\nabla \varphi|^{2}+\beta}}-\frac{\alpha \sum{ }_{i}\left(\sum \varphi_{j} \varphi_{j i}\right)^{2}}{\left(|\nabla \varphi|^{2}+\beta\right)^{3 / 2}} \\
& -\alpha^{\prime} \sqrt{|\nabla \varphi|^{2}+\beta}-\frac{\alpha \beta^{\prime}}{2 \sqrt{|\nabla \varphi|^{2}+\beta}}-c_{t}+\Delta c-2 \sum \varphi_{i} c_{i}+\sum f_{i} c_{i} .
\end{aligned}
$$

Suppose $\psi<0$ when $t=0$. Assume that at $t_{0}>0, \psi$ becomes zero at some point in the interior of the manifold and $\psi<0$ for $t<t_{0}$. Then $\psi_{t} \geq 0, \nabla \psi=0$ and $\Delta \psi \leq 0$ at this point. Hence

$$
\begin{aligned}
(2- & \left.\frac{\alpha}{\sqrt{|\nabla \varphi|^{2}+\beta}}\right) \sum_{i, j}\left[\varphi_{i j}-\frac{1}{2}\left(2-\frac{\alpha}{\sqrt{|\nabla \varphi|^{2}+\beta}}\right)^{-1}\left(f_{i, j}+f_{j, i}\right)\right]^{2} \\
\leq & \frac{1}{4}\left(2-\frac{\alpha}{\sqrt{|\nabla \varphi|^{2}+\beta}}\right)^{-1} \sum_{i, j}\left(f_{i, j}+f_{j, i}\right)^{2} \\
& +\sum\left(\Delta f_{i}-2 c_{i}\right) \varphi_{i}+\Delta V-\frac{-\alpha\left(\sum f_{i, j} \varphi_{i} \varphi_{j}+\sum \varphi_{i} V_{i}\right)}{\sqrt{|\nabla \varphi|^{2}+\beta}} \\
& -2 \sum R_{i j} \varphi_{i} \varphi_{j}+\sum R_{i j} f_{i} \varphi_{j}-\frac{\left(\alpha \sum_{i}\left(\sum_{j} \varphi_{j} \varphi_{j i}\right)^{2}\right.}{\left(|\nabla \varphi|^{2}+\beta\right)^{3 / 2}} \\
& -\alpha^{\prime} \sqrt{|\nabla \varphi|^{2}+\beta}-\frac{\alpha \beta^{\prime}}{\sqrt[2]{|\nabla \varphi|^{2}+\beta}}-c_{t}+\Delta c+\sum f_{i} c_{i} .
\end{aligned}
$$


By the arithmetic-geometric inequality, the lefthand side is greater than

$$
\left(2-\frac{\alpha}{\sqrt{|\nabla \varphi|^{2}+\beta}}\right) n^{-1}\left[\Delta \varphi-\left(2-\frac{\alpha}{\sqrt{|\nabla \varphi|^{2}+\beta}}\right)^{-1} \sum f_{i, i}\right]^{2} .
$$

Note that at $\psi=0, \Delta \varphi=\alpha \sqrt{|\nabla \varphi|^{2}+\beta}+c$.

If we assume that for some $a$, the following inequality holds:

$$
2 \Sigma R_{i j} x_{i} x_{j}-\sum R_{i j} f_{i} x_{j}-\sum\left(\Delta f_{i}-\left(\sum f_{j, j}\right)_{i}\right) x_{i} \geq-a
$$

for all $x$, then we can take $\alpha=0$ in the above inequalities and take

$$
c=\frac{n}{2 t}+\frac{1}{2} \sum f_{j, j}+b
$$

to conclude

$$
\begin{aligned}
\frac{2}{n}\left(\frac{n}{2 t}+b\right)^{2} & \leq \frac{1}{8} \sum\left(f_{i, j}+f_{j, i}\right)^{2}+a+\Delta V \\
+\frac{n}{2 t^{2}} & -\frac{1}{2}\left(\sum f_{j, j}\right)_{t}+\Delta\left(\frac{1}{2} \sum f_{j, j}\right)+\sum f_{i}\left(\frac{1}{2} \sum f_{j, j}\right)_{i} .
\end{aligned}
$$

This inequality is violated if

$$
\begin{aligned}
\frac{2 b}{t}+\frac{2}{n} b^{2} & >\frac{1}{8} \sum\left(f_{i, j}+f_{j, i}\right)^{2}+a+\Delta V \\
& -\frac{1}{2}\left(\sum f_{j, j}\right)_{t}+\Delta\left(\frac{1}{2} \sum f_{j, j}\right)+\sum f_{i}\left(\frac{1}{2} \sum f_{j, j}\right)_{i} .
\end{aligned}
$$

On a noncompact manifold, $\psi$ may not achieve its maximum in the interior of the manifold. In that case, we choose a nonnegative function $\rho$ which tends to infinity when $r$ tends to infinity. If $\psi$ is bounded, the function $\psi-\epsilon \rho$ then attains a maximum somewhere for $t \leq t_{0}$. At such a point, $\Delta \psi \leq \epsilon \Delta \rho$ and $\nabla \psi=\epsilon \nabla \rho$. Hence

$$
\begin{aligned}
-\epsilon \Delta \rho & \leq-2 \epsilon \sum \varphi_{i} \rho_{i}-\epsilon \sum f_{i} \rho_{i}-2 \sum \varphi_{i j}^{2}+2 \sum f_{i, j} \varphi_{i j} \\
& +\sum\left(\Delta f_{i}\right) \varphi_{i}+\Delta V-\frac{\left(\alpha\left(\sum f_{i j} \varphi_{i} \varphi_{j}+\sum \varphi_{i} V_{i}\right)\right.}{\sqrt{|\nabla \varphi|^{2}+\beta}} \\
& -2 \sum R_{i j} \varphi_{i} \varphi_{j}+\frac{\alpha \sum \varphi_{i j}^{2}}{\sqrt{|\nabla \varphi|^{2}+\beta}}-\frac{\alpha \sum_{i}\left(\sum_{j} \varphi_{j} \varphi_{j i}\right)^{2}}{\left(|\nabla \varphi|^{2}+\beta\right)^{3 / 2}} \\
& -\alpha^{\prime} \sqrt{|\nabla \varphi|^{2}+\beta}-\frac{\alpha \beta^{\prime}}{2 \sqrt{|\nabla \varphi|^{2}+\beta}}-c_{t}+\Delta c \\
& -2 \sum \varphi_{i} c_{i}+\sum f_{i} c_{i} .
\end{aligned}
$$



that

In this case we can take $c=\frac{n}{2 t}+\frac{1}{2} \sum f_{j, j}+b$. Then under the assumption

$$
2 \sum R_{i j} x_{j} x_{j}-\sum R_{i j} f_{i} x_{j}-\sum\left(\Delta f_{i}-\left(\sum f_{j, j}\right)_{i}-2 \epsilon \rho_{i}\right) x_{i} \geq-a
$$

for all $x_{i}$. We find

$$
\begin{aligned}
-\epsilon \Delta \rho+ & \frac{2}{n}\left(\frac{n}{2 t}+b\right)^{2} \leq-\epsilon \sum f_{i} \rho_{i}+\frac{1}{8} \sum\left(f_{i, j}+f_{j, i}\right)^{2}+a+\Delta V \\
+ & \frac{n^{2}}{4 t^{2}}-\frac{1}{2}\left(\sum f_{i, i}\right)_{t}+\Delta\left(\frac{1}{2} \sum f_{i, i}\right)+\sum f_{i}\left(\frac{1}{2} \sum f_{j, j}\right)_{i} .
\end{aligned}
$$

We can choose $\rho$ to be of the form $\log \left(d^{2}+1\right)$ where $d$ is the distance function. Hence if we assume that $\left(d^{2}+1\right)^{-1}\left(\sum f_{i}^{2}\right)$ is bounded for all $t$, we can let $\epsilon \rightarrow 0$ in (1.13) and obtain

$$
\begin{aligned}
& \frac{2}{n}\left(\frac{n}{2 t}+b\right)^{2} \leq \frac{1}{8} \sum\left(f_{i, j}+f_{j, i}\right)^{2}+a+\Delta V \\
&+\frac{n}{2 t^{2}}-\frac{1}{2}\left(\sum f_{i, i}\right)_{t}+\Delta\left(\frac{1}{2} \sum f_{i, i}\right)+\sum f_{i}\left(\frac{1}{2} \sum f_{j, j}\right)_{i} .
\end{aligned}
$$

If we also assume that $\left(d^{2}+1\right)^{-1}|\nabla \varphi|^{2}$ is bounded (in space) for all $t>0$, then we can let $\epsilon \rightarrow 0$.

By a parametrix argument, if both $\left(d^{2}+1\right)^{-1} \sum f_{i}^{2}$ and $\left(d^{2}+1\right)^{-1}|V|$ in (1.12) are bounded, then both $\left(d^{2}+1\right)^{-1}|\nabla \varphi|^{2}$ and $\psi$ are bounded for small time. Hence we have proved the following

Theorem 1. Let $u$ be a positive solution of (1.1) so that $\left(d^{2}+1\right)^{-1} \sum f_{i}^{2}$ and $\left(d^{2}+1\right)^{-1} V$ are bounded. Suppose there is a constant a so that

$$
2 \sum R_{i j} x_{i} x_{j}-\sum R_{i j} f_{i} x_{j}-\sum\left(\Delta f_{i}-\left(\sum f_{i, j}\right)_{i}\right) x_{j} \geq-a
$$

for all $x_{i}$. Suppose that for some constant $b$

$$
\begin{aligned}
\frac{2}{n} b^{2}+\frac{2 b}{t} & >\frac{1}{8} \sum\left(f_{i, j}+f_{j, i}\right)^{2}+a+\Delta V \\
& -\frac{1}{2}\left(\sum f_{i, i}\right)_{t}+\Delta\left(\frac{1}{2} \sum f_{i, i}\right)+\sum f_{i}\left(\frac{1}{2} \sum f_{j, j}\right)_{i}
\end{aligned}
$$

Then $-(\log u)_{t}+|\nabla \log u|^{2}+\sum f_{i}(\log u)_{i}-V-\frac{n}{2 t}-b-\frac{1}{2} \sum f_{i, i}$ is nonpositive for all $t>0$ if it is nonpositive for $0 \leq t \leq \epsilon$ for some $\epsilon>0$. 
Note that the last assumption can be checked by the parametrix argument.

Let us now consider the case when the manifold is compact with boundary. In this case, we shall assume that the vector field $F=\sum f_{i} \frac{\partial}{\partial x_{i}}$ is tangential to the boundary. We require that $u$ is constant on the boundary.

If $\psi$ achieves its maximum on the boundary, then $\frac{\partial \psi}{\partial \nu} \geq 0$ at that point. On the other hand, $u$ is constant implies that $\frac{d \varphi}{d t}=0$ on the boundary. Hence $\frac{d}{d t} \frac{\partial \varphi}{\partial \nu}=0$ and

$$
\begin{aligned}
0 \leq \frac{\partial \psi}{\partial \nu} & =\frac{d}{d t} \frac{\partial \varphi}{\partial \nu}+\frac{\partial}{\partial \nu}\left(|\nabla \varphi|^{2}\right)-\frac{\partial}{\partial \nu} F(\varphi)-\frac{\partial}{\partial \nu}(V)-\frac{\partial}{\partial \nu}(c) \\
& =\frac{\partial}{\partial \nu}\left(|\nabla \varphi|^{2}\right)-\frac{\partial}{\partial \nu}\left(V+\frac{1}{2} \sum_{i} f_{i, i}\right)
\end{aligned}
$$

Let us now assume the boundary of the manifold has positive mean curvature. Then $\frac{\partial}{\partial \nu}\left(|\nabla \varphi|^{2}\right) \leq 0$. Hence if we assume $\frac{\partial}{\partial \nu}\left(V+\frac{1}{2} \sum_{i} f_{i, i}\right) \geq 0$, we can prove the following

Theorem 2. Let $u$ be a positive solution of (1.1) which satisfies the condition that $u=$ constant on the boundary. Assume that the boundary has positive mean curvature and $\frac{\partial}{\partial \nu}\left(V+\frac{1}{2} \sum f_{i, i}\right) \geq 0$. Assume that $(1.15)$ and (1.16) hold for some constants $a$ and $b$. Then

$(1.18)-(\log u)_{t}+|\nabla \log u|^{2}+\sum f_{i}(\log u)_{i}-V-\frac{n}{2 t}-b-\frac{1}{2} \sum f_{i, i} \leq 0$.

Let us now explain (1.15) and (1.16). If $f_{i}$ is a gradient vector field and $R_{i j}=0$ (as in Euclidean space, (1.15) automatically holds. It is remarkable that in (1.16), only $\Delta V$ appears. In particular, if $V$ is linear for each time and $\Delta V=0$, the theorem applies.

Let us now consider the case when (1.15) does not hold for any choice of $a$. We shall take $c=\frac{n}{2 t}+b$ where $b$ is a positive constant to be determined. We shall choose $\beta=d+\frac{e}{t}$ where $d$ and $e$ are positive constants to be chosen. Choose $d \geq \alpha^{2}$ so that $\alpha\left(\sqrt{|\nabla \varphi|^{2}+\beta}\right)^{-1} \leq 1$. 
The previous argument requires that the following inequality be violated:

$$
\begin{gathered}
\left(2-\frac{\alpha}{\sqrt{\left(|\nabla \varphi|^{2}+\beta\right.}}\right) n^{-1}\left[\alpha \sqrt{|\nabla \varphi|^{2}+\beta}+\frac{n}{2 t}+b\right. \\
\left.-\left(2-\frac{\alpha}{\sqrt{\left(|\nabla \varphi|^{2}+\beta\right.}}\right)^{-1} \sum f_{i, i}\right]^{2} \\
\leq \frac{1}{4}\left(2-\frac{\alpha}{\sqrt{\left(|\nabla \varphi|^{2}+\beta\right.}}\right)^{-1} \sum_{i, j}\left(f_{i, j}+f_{j, i}\right)^{2}+\sum\left(\Delta f_{i}\right) \varphi_{i}+\Delta V \\
-\alpha \frac{\left(\sum f_{i, j} \varphi_{i} \varphi_{j}+\sum \varphi_{i} V_{i}\right)}{\sqrt{|\nabla \varphi|^{2}+\beta}}-2 \sum R_{i j} \varphi_{i} \varphi_{j}+2 \sum R_{i j} f_{i} \varphi_{j} \\
+\frac{\alpha e}{2 t^{2} \sqrt{|\nabla \varphi|^{2}+\beta}}+\frac{n}{2 t^{2}} .
\end{gathered}
$$

The righthand side is not greater than

$$
\begin{aligned}
& \frac{1}{4} \sum_{i, j}\left(f_{i, j}+f_{j, i}\right)^{2}+\Delta V-\alpha|\nabla V|+\epsilon|\nabla \varphi|^{2}+\frac{1}{4 \epsilon} \sum_{i}\left(\Delta f_{i}\right)^{2} \\
& +\epsilon|\nabla \varphi|^{2}+\frac{\alpha^{2}}{16 \epsilon} \sum_{i, j}\left(f_{i, j}+f_{j, i}\right)^{2}+\epsilon|\nabla \varphi|^{2}+\frac{1}{\epsilon} \sum_{j}\left(\sum_{i} R_{i j} f_{i}\right)^{2} \\
& -2 \sum R_{i j} \varphi_{i} \varphi_{j}+\frac{\alpha e}{2 t^{2} \sqrt{|\nabla \varphi|^{2}+\beta}}+\frac{n}{2 t^{2}}
\end{aligned}
$$

where $\epsilon>0$ is arbitrary. If $b \geq\left|\Sigma f_{i, i}\right|$, the lefthand side is not less than

$$
\begin{gathered}
\frac{\alpha^{2}}{n}\left(|\nabla \varphi|^{2}+\beta\right)+\frac{n}{2 t^{2}}-\frac{\alpha n}{4 t^{2} \sqrt{|\nabla \varphi|^{2}+\beta}} \\
\frac{\alpha \sqrt{|\nabla \varphi|^{2}+\beta}}{t}+\left(b-\sum f_{i, i}\right)^{2} .
\end{gathered}
$$

By choosing $e \geq \frac{n}{2}$, we can arrange

$$
\frac{\alpha \sqrt{|\nabla \varphi|^{2}+\beta}}{t}-\frac{\alpha n}{4 t^{2} \sqrt{|\nabla \varphi|^{2}+\beta}} \geq \frac{\alpha e}{2 t^{2} \sqrt{|\nabla \varphi|^{2}+\beta}} .
$$

Hence we simply choose $\alpha$ and $b$ so that

$$
\frac{\alpha^{2}}{n}|\nabla \varphi|^{2} \geq 3 \epsilon|\nabla \varphi|^{2}-2 \sum R_{i j} \varphi_{i} \varphi_{j}
$$




$$
\begin{gathered}
\left(b-\sum f_{i, i}\right)^{2}>\left(\frac{1}{4}+\frac{\alpha^{2}}{16 \epsilon}\right) \sum_{i, j}\left(f_{i, j}+f_{j, i}\right)^{2}+\Delta V-\alpha|\nabla V| \\
+\frac{1}{4 \epsilon} \sum_{i}\left(\Delta f_{i}\right)^{2}+\frac{1}{\epsilon} \sum_{j}\left(\sum_{i} R_{i j} f_{i}\right)^{2} \\
b \geq\left|\sum_{i} f_{i, i}\right| .
\end{gathered}
$$

Theorem 3. Let $u$ be a positive solution of (1.1) so that $\left(d^{2}+1\right)^{-1} \sum_{i} f_{i}^{2}$ and $\left(d^{2}+1\right)^{-1} V$ are bounded. Suppose that we can choose constants $\alpha$ and $b$ so that (1.23) and (1.24) hold. Then

$$
\begin{aligned}
& -(\log u)_{t}+|\nabla \log u|^{2}+\sum f_{i}(\log u)_{i}-V \\
& \leq \alpha \sqrt{|\nabla(\log u)|^{2}+d+\frac{e}{t}}+\frac{n}{2 t}+b
\end{aligned}
$$

where $d \geq \alpha^{2}$ and $e \geq \frac{n}{2}$.

\section{Estimate on the time derivative}

In this section, we shall make use of the estimate of the previous section. Let

$$
\psi=\varphi+t \varphi_{t}+F
$$

where $F$ is a function to be determined.

Then

$$
\begin{aligned}
\frac{\partial \psi}{\partial t}-\Delta \psi= & \left(\varphi_{t}-\Delta \varphi\right)+\varphi_{t}+t\left(\varphi_{t}-\Delta \varphi\right)_{t}+F_{t}-\Delta F \\
= & \left(-|\nabla \varphi|^{2}+\sum f_{i} \varphi_{i}+V\right)+\varphi_{t} \\
& +t\left(-|\nabla \varphi|^{2}+\sum f_{i} \varphi_{i}+V\right)_{t}+F_{t}-\Delta F \\
= & -2 \varphi_{i}\left(t \varphi_{t}+\varphi+F\right)_{i} \\
& +\sum f_{i}\left(t \varphi_{t}+\varphi+F\right)_{i}+\varphi_{t} \\
& +|\nabla \varphi|^{2}+\sum f_{i} \varphi_{i}+V+t V_{t}+2 \sum F_{i} \varphi_{i} \\
& +t \sum\left(f_{i}\right)_{t} \varphi_{i}-\sum f_{i} F_{i}+F_{t}-\Delta F
\end{aligned}
$$


The first simple case is to assume $f_{i}$ is a gradient vector field and $R_{i j}=0$. In this case we choose $F$ so that $t\left(f_{i}\right)_{t}+f_{i}=-2 F_{i}, a$ can be chosen to be zero in (1.8) and $b>0$ can be chosen so that

$$
\begin{aligned}
\frac{2}{n} b^{2} & =\frac{1}{8} \sum_{i, j}\left(f_{i, j}+f_{j, i}\right)^{2}+\Delta V \\
& +\frac{1}{2} \Delta\left(\sum_{i} f_{i, i}\right)-\frac{1}{2}\left(\sum f_{i, i}\right)_{t}+\sum f_{i}\left(\frac{1}{2} \sum f_{j, j}\right)_{i} .
\end{aligned}
$$

Then by Theorem 1 ,

$$
\varphi_{t}+|\nabla \varphi|^{2}-\sum f_{i} \varphi_{i}-V-\frac{n}{2 t}-b-\frac{1}{2} \sum f_{i, i} \leq 0 .
$$

Hence

$$
\begin{aligned}
& \varphi_{t}+|\nabla \varphi|^{2}+V+t V_{t}+\sum\left(2 F_{i}+t\left(f_{i}\right)_{t}+f_{i}\right) \varphi_{i}-\sum f_{i} F_{i}+F_{t}-\Delta F \\
& \leq 2 V+\frac{n}{2 t}+b+\sum f_{i, i}+t V_{t}+\frac{1}{4} t\left(\sum f_{i}^{2}\right)_{t}+\frac{1}{2} \sum f_{i}^{2}+F_{t} .
\end{aligned}
$$

If we replace $F$ by $-\frac{n}{2} \log t+F$, we obtain the following:

Theorem 4. Assume that in Theorem $1, R_{i j}=0$ and $\left(f_{i}\right)$ is a gradient vector field. Then if $u$ is a positive solution of (1.1) and $b>0$ is defined by (2.3), then

$$
-t(\ln u)_{t}-\log u-\frac{n}{2} \ln t-c t+F \leq 0
$$

such that $t\left(f_{i}\right)_{t}+f_{i}=-2 F_{i}$, where

$$
c=\sup \left(2 V+b+\frac{1}{2} \sum_{i} f_{i, i}+t V_{t}+\frac{1}{2} \sum f_{i}^{2}+\frac{1}{4} t\left(\sum f_{i}^{2}\right)_{t}+F_{t}\right),
$$

and

$$
\lim _{t \rightarrow 0}\left(-t(\ln u)_{t}-\log u-\frac{n}{2} \ln t+F\right) \leq 0 .
$$

Note that when $f_{i}=0$, we need only make the assumption $R_{i j} \geq 0$ instead of $R_{i j}=0$. 
Corollary. Under the assumption of Theorem 4,

$$
t \log u-\lim _{\tau \rightarrow 0} \tau \log u(\tau) \geq-\frac{n}{2} t \log t+\frac{n}{2} t-\frac{c t^{2}}{2}+\int_{0}^{t} F
$$

and

$$
u \geq t^{-n / 2} \exp \left[\frac{1}{t} \int_{0}^{t} F+\frac{n}{2}+\frac{1}{t} \lim _{\tau \rightarrow 0}(\tau \log u(\tau))-\frac{c t}{2}\right] .
$$

When $f_{i}$ is not a gradient vector field, the estimate is more complicated. However, the opposite case when $f_{i, j}+f_{j, i}=0$ is quite simple. (This corresponds to the vector field defined by a one-parameter family of orthogonal matrices.)

In this case, let

$$
\psi=\varphi+t \varphi_{t}-t \sum_{i} f_{i} \varphi_{i}-\frac{n}{2} \ln t+F(t) .
$$

Then

$$
\begin{aligned}
\psi_{t}-\Delta \psi= & \varphi_{t}-\Delta \varphi+\varphi_{t}+t\left(\varphi_{t}-\Delta \varphi\right)_{t}-\sum f_{i} \varphi_{i}-t \sum\left(f_{i}\right)_{t} \varphi_{i} \\
& +t \sum R_{i j} f_{i} \varphi_{j}-t \sum f_{i}\left(\varphi_{t}-\Delta \varphi\right)_{i} \\
& +2 t \sum f_{i, j} \varphi_{i j}+t \sum \Delta f_{i} \varphi_{i}-\frac{n}{2 t}+F^{\prime} \\
= & \left(-|\nabla \varphi|^{2}+\sum f_{i} \varphi_{i}+V\right)+\varphi_{t} \\
& +t\left(-|\nabla \varphi|^{2}+\sum f_{i} \varphi_{i}+V\right)_{t}-\sum f_{i} \varphi_{i} \\
& -t \sum\left(f_{i}\right)_{t} \varphi_{i}+t \sum R_{i j} f_{i} \varphi_{j} \\
& -t \sum f_{i}\left(-|\nabla \varphi|^{2}+\sum f_{i} \varphi_{i}+V\right)_{i} \\
& +2 t \sum f_{i, j} \varphi_{i j}+t \sum \Delta f_{i} \varphi_{i}-\frac{n}{2 t}+F^{\prime} \\
= & \varphi_{t}-2 \sum \varphi_{i} \psi_{i}+|\nabla \varphi|^{2}-2 t \sum f_{i, j} \varphi_{i} \varphi_{j} \\
& -\sum f_{i} \varphi_{i}+V+t V_{t}+\sum f_{i} \psi_{i}+t \sum R_{i j} f_{i} \varphi_{j} \\
& +2 t \sum f_{i, j} \varphi_{i j}+t \sum\left(\Delta f_{i}\right) \varphi_{i}-\frac{n}{2 t}+F^{\prime} .
\end{aligned}
$$

Note that $f_{i j}=-f_{j, i}$ implies $\sum f_{i, j} \varphi_{i} \varphi_{j}=0=\sum f_{i, j} \varphi_{i j}$. If $R_{i j}=0$, $\Delta f_{i}=0$ also. Theorem 1 says that

$$
\varphi_{t}+|\nabla \varphi|^{2}-\sum \varphi_{i} f_{i}-V-\frac{n}{2 t} \leq \sqrt{\frac{n}{2}(\Delta V)_{+}} .
$$


Hence when $\psi_{i}=0(2.11)$ is less than

$$
2 V+t V_{t}+\sqrt{\frac{n}{2}(\Delta V)_{+}}+F^{\prime}
$$

Theorem 5. Assume that in Theorem $1, R_{i j}=0$ and $f_{i, j}=-f_{j, i}$. Let $u$ be a positive solution of (1.1). Let $F$ be a function of $t$ so that

$$
2 V+t V_{t}+\sqrt{\frac{n}{2}(\Delta V)_{+}}+F^{\prime} \leq 0 .
$$

and $\lim _{t \rightarrow 0}\left[-t(\log u)_{t}-\log u+\sum f_{i}(\log u)_{i}-\frac{n}{2} \log t+F\right] \leq 0$. Then

$(2.14)-t(\log u)_{t}-\log u+\sum f_{i}(\log u)_{i}-\frac{n}{2} \log t+F \leq 0 \quad$ for all $t$.

If $f_{i}$ is neither a gradient vector field nor a Killing field (i.e., $f_{i, j}=-f_{j, i}$ ), we proceed as follows. Let

$$
\psi=\varphi+t \varphi_{t}-\gamma(t) \sqrt{|\nabla \varphi|^{2}+d+\frac{e}{t}}-\frac{n}{2} \ln t+F(t) .
$$

Then

$$
\begin{aligned}
\psi_{t}- & \Delta \psi=\varphi_{t}-\Delta \varphi+\varphi_{t}+t\left(\varphi_{t}-\Delta \varphi\right)_{t} \\
& -\gamma^{\prime} \sqrt{|\nabla \varphi|^{2}+d+\frac{e}{t}}+F^{\prime}-\gamma\left(\sum R_{i j} \varphi_{i} \varphi_{j}\right)\left(|\nabla \varphi|^{2}+d+\frac{e}{t}\right)^{-\frac{1}{2}} \\
& -\gamma\left[\sum_{i} \varphi_{i}\left(\varphi_{t}-\Delta \varphi\right)_{i}-\frac{e}{t^{2}}\right]\left(|\nabla \varphi|^{2}+d+\frac{e}{t}\right)^{-1 / 2} \\
& -\gamma\left(\sum_{i j}^{2}\right)\left(|\nabla \varphi|^{2}+d+\frac{e}{t}\right)^{-1 / 2} \\
& +\gamma \sum_{j}\left(\sum_{i} \varphi_{i} \varphi_{i j}\right)^{2}\left[|\nabla \varphi|^{2}+d+\frac{e}{t}\right]^{-3 / 2}-\frac{n}{2 t} \\
= & -|\nabla \varphi|^{2}+\sum_{i} f_{i}+V+\varphi_{t} \\
& +t\left(-|\nabla \varphi|^{2}+\sum_{i} f_{i}+V\right)_{t}-\gamma^{\prime} \sqrt{|\nabla \varphi|^{2}+d+\frac{e}{t}}+F^{\prime} \\
& -\gamma\left[\sum_{i} \varphi_{i}\left(\left(-|\nabla \varphi|^{2}+\sum f_{i} \varphi_{i}+V\right)_{i}-\frac{e}{t^{2}}\right]\left[|\nabla \varphi|^{2}+d+\frac{e}{t}\right]^{-1 / 2}\right. \\
& -\gamma\left(\sum_{i j} R_{i} \varphi_{j}\right)\left(|\nabla \varphi|^{2}+d+\frac{e}{t}\right)^{-1 / 2} \\
& -\gamma\left(\sum_{\left.\varphi_{i j}^{2}\right)\left(|\nabla \varphi|^{2}+d+\frac{e}{t}\right)^{-1 / 2}}\right. \\
+ & \gamma \sum_{j}\left(\sum_{i} \varphi_{i} \varphi_{i j}\right)^{2}\left(|\nabla \varphi|^{2}+d+\frac{e}{t}\right)^{-3 / 2}-\frac{n}{2 t}
\end{aligned}
$$




$$
\begin{aligned}
= & \varphi_{t}-2 \sum_{i} \varphi_{i} \psi_{i}+|\nabla \varphi|^{2}+V+t V_{t}+\sum f_{i} \psi_{i}+F^{\prime} \\
& -\gamma\left[\sum f_{i, j} \varphi_{i} \varphi_{j}+\sum \varphi_{i} V_{i}-\frac{e}{t^{2}}\right]\left(|\nabla \varphi|^{2}+d+\frac{e}{t}\right)^{-1 / 2} \\
& -\gamma^{\prime} \sqrt{|\nabla \varphi|^{2}+d+\frac{e}{t}} \\
& -\gamma\left[\sum R_{i j} \varphi_{i} \varphi_{j}+\sum \varphi_{i j}^{2}\right]\left(|\nabla \varphi|^{2}+d+\frac{e}{t}\right)^{-1 / 2} \\
& +\gamma \sum_{j}\left(\sum_{i} \varphi_{i} \varphi_{i j}\right)^{2}\left[|\nabla \varphi|^{2}+d+\frac{e}{t}\right)^{-3 / 2}+t \sum\left(f_{i}\right)_{t} \varphi_{i}-\frac{n}{2 t} \\
\leq & -2 \sum_{i} \varphi_{i} \psi_{i}+\sum f_{i} \psi_{i}+2 V+t V_{t} \\
& +\frac{1}{2} \sum_{i} f_{i, i}+\sum\left(t\left(f_{i}\right)_{t}+f_{i}\right) \varphi_{i}+\alpha \sqrt{|\nabla \varphi|^{2}+d+\frac{e}{t}} \\
= & b F^{\prime}+\gamma\left(\frac{1}{2} \sqrt{\sum\left(f_{i, j}+f_{j, i}\right)^{2}}|\nabla \varphi|+|\nabla V|+\frac{\sqrt{e}}{t}\right) \\
& -\gamma^{\prime} \sqrt{|\nabla \varphi|^{2}+d+\frac{e}{t}}-\gamma\left(R_{i j}\right)_{-}|\nabla \varphi|,
\end{aligned}
$$

where $\left(R_{i j}\right)_{-}$is the smallest eigenvalue of the Ricci curvature $R_{i j}$.

Theorem 6. Using the notation of Theorem 3, we choose a function $\gamma(t)$ so that

$$
\begin{aligned}
& \left(-\gamma^{\prime}+\alpha\right) \sqrt{|\nabla \varphi|^{2}+d+\frac{e}{t}}+\sum\left(t\left(f_{i}\right)_{t}+f_{i}\right) \varphi_{i} \\
& +\gamma\left(\frac{1}{2} \sqrt{\sum\left(f_{i, j}+f_{j, i}\right)^{2}}|\nabla \varphi|+|\nabla V|+\frac{\sqrt{e}}{t}\right)-\gamma\left(R_{i j}\right)_{-}|\nabla \varphi| \leq 0 .
\end{aligned}
$$

We also choose $F(t)$ so that

$$
2 V+t V_{t}+b+F^{\prime} \leq 0 .
$$

Then under the same assumption as in Theorem 3,

$$
-\log u-t(\log u)_{t}-\gamma \sqrt{|\nabla \varphi|^{2}+d+\frac{e}{t}}-\frac{n}{2} \ln t+F(t) \leq 0 .
$$




\section{References}

1. S. Y. Cheng, P. Li and S.-T. Yau, On the upper estimate of the heat kernel of a complete Riemannian manifold, Amer. J. Math. 10-3, 1021-1063.

2. P. Li and S.-T. Yau, On the parabolic kernel of the Schrödinger operator, Acta Math. 156, 153-201.

Department of Mathematics, Harvard University, Cambidge, Ma 02138

E-mail address: yau@math.harvard.edu 